\title{
Short-course regimens of rifapentine plus isoniazid to treat latent tuberculosis infection in older Chinese patients: a randomised controlled study
}

\author{
Lei Gao ${ }^{1,5,6}$, Haoran Zhang ${ }^{1,5}$, Henan Xin ${ }^{1,5}$, Jianmin Liü ${ }^{2,5}$, Shouguo Pan ${ }^{3,5}$, \\ Xiangwei $\mathrm{Li}^{1}$, Ling Guan ${ }^{2}$, Fei Shen ${ }^{2}$, Zisen Liu ${ }^{3}$, Dakuan Wang ${ }^{3}$, Xueling Guan ${ }^{2}$, \\ Jiaoxia Yan ${ }^{3}$, Hengjing $\mathrm{Li}^{1}$, Boxuan Feng ${ }^{1}$, Xuefang Cao ${ }^{1}, \mathrm{Yu} \mathrm{Chen}^{2}$, Wei Cui ${ }^{2}$, \\ Zongde Zhang ${ }^{4}$, Yu Ma ${ }^{4}$, Xiaoyou Chen ${ }^{4}$, Xinhua Zhou ${ }^{4}$ and Qi Jin ${ }^{1,6}$ for the \\ LATENTTB-NSTM study team
}

@ERSpublications

A randomised controlled trial suggested that the short regimens tested for treatment of latent tuberculosis infection must be used with caution among the elderly because of the high rates of adverse effects http://ow.ly/AmmM30m7bYd

Cite this article as: Gao L, Zhang $\mathrm{H}$, Xin $\mathrm{H}$, et al. Short-course regimens of rifapentine plus isoniazid to treat latent tuberculosis infection in older Chinese patients: a randomised controlled study. Eur Respir J 2018; 52: 1801470 [https://doi.org/10.1183/13993003.01470-2018].

ABSTRACT Latent tuberculosis infection (LTBI) management is now a critical component of the World Health Organization's End TB Strategy.

In this randomised controlled trial (Chinese Clinical Trial Registry identifier ChiCTR-IOR-15007202), two short-course regimens with rifapentine plus isoniazid (a 3-month once-weekly regimen and a 2-month twice-weekly regimen) were initially designed to be evaluated for rural residents aged 50-69 years with LTBI in China.

Due to the increasingly rapid growth and unexpected high frequency of adverse effects, the treatments were terminated early (after 8 weeks for the once-weekly regimen and after 6 weeks for the twice-weekly regimen). In the modified intention-to-treat analysis on the completed doses, the cumulative rate of active disease during 2 years of follow-up was $1.21 \%$ ( 14 out of 1155 ) in the untreated controls, $0.78 \%$ (10 out of 1284) in the group that received the 8 -week once-weekly regimen and $0.46 \%$ (six out of 1299) in the group that received the 6-week twice-weekly regimen. The risk of active disease was decreased, with an adjusted hazard ratio of 0.63 (95\% CI $0.27-1.43)$ and 0.41 (95\% CI 0.15-1.09) for the treatments, respectively. No significant difference was found in the occurrence of hepatotoxicity (1.02\% (13 out of 1279 ) versus $1.17 \%$ ( 15 out of 1279$) ; \mathrm{p}=0.704$ ).

The short regimens tested must be used with caution among the elderly because of the high rates of adverse effects. Further work is necessary to test the ultrashort regimens in younger people with LTBI.

This article has supplementary material available from erj.ersjournals.com

This study is registered at www.chictr.org.cn with identifier ChiCTR-IOR-15007202. The corresponding author can provide, upon request, individual participant data that underlie the results reported in this article (except variables, if any, that may allow identification of patients), after applying necessary measures to guarantee that no individual is identified or identifiable.

Received: Aug 022018 | Accepted after revision: Sept 242018

Copyright $\odot$ ERS 2018 
Affiliations: ${ }^{1} \mathrm{MOH}$ Key Laboratory of Systems Biology of Pathogens, Institute of Pathogen Biology and Center for Tuberculosis, Chinese Academy of Medical Sciences and Peking Union Medical College, Beijing, China. ${ }^{2}$ The Sixth People's Hospital of Zhengzhou, Zhengzhou, China. ${ }^{3}$ Center for Diseases Control and Prevention of Zhongmu, Zhongmu, China. ${ }^{4}$ Beijing Chest Hospital, Capital Medical University, Beijing Key Laboratory for Drug Resistant Tuberculosis Research, Beijing Tuberculosis and Thoracic Tumor Research Institute, Beijing, China. ${ }^{5}$ These authors contributed equally to this work. ${ }^{6}$ These authors contributed equally to this work.

Correspondence: Qi Jin, Institute of Pathogen Biology, Chinese Academy of Medical Sciences, No. 9 Dong Dan San Tiao, Beijing 100730, China. E-mail: jinqidipbcams.ac.cn

\section{Introduction}

Close to a quarter of the global population is latently infected with Mycobacterium tuberculosis; millions of active tuberculosis (TB) cases might emerge from this reservoir with a lifetime risk of TB of 5-10\% [1-3]. Prevention of disease development through treatment of latent TB infection (LTBI) is now a critical component of the World Health Organization (WHO)'s End TB Strategy [4-6]. China is a high-burden country in terms of both active TB and LTBI, but no national guidelines on LTBI management have yet been developed. Only targeting the high-risk populations recommended by the WHO guidelines, e.g. people living with HIV and close household contacts, for LTBI management might not achieve a significant decline in the incidence of TB because these populations contribute only a small minority of active TB cases in China [7, 8]. For example, in 2017, only $1 \%$ of incident TB cases were HIV-positive in China compared with $10 \%$ in America and Europe and $60 \%$ in South Africa [1]. Therefore, in order to reduce the incidence of $\mathrm{TB}$, selecting at-risk populations for LTBI management should adapt to the national epidemiology of TB and other local determinants $[5,6]$.

Our observational studies reported that half of new infections and three-quarters of active disease occur among individuals aged $>50$ years in the general population in China [7, 9, 10]. Modelling analysis indicated that preventative therapy in the elderly would be the most effective single intervention to accelerate the decline in the incidence of $\mathrm{TB}$ in China if it could be made feasible by managing the relatively high risks of hepatic adverse events [11-14]. Nevertheless, in our view, it is still a theoretical exploration to extend LTBI management to all elderly populations in high-burden countries like China. It is a major challenge to balance the benefits and risks of LTBI treatment in the elderly at the public health level. Targeting subgroups in the elderly at higher risk of developing active disease, such as those with a history of prior $\mathrm{TB}$, would be meaningful and feasible in the current situation. Our previous report proposed that the incidence of TB might decline by $30 \%$ in rural communities if effective LTBI intervention is provided for those individuals with prior TB and $>50$ years old [7]. This accounts for only $3 \%$ of the rural population, while it largely improves the cost-efficacy of the targeted intervention at a community level.

In addition, with doubt, short-course regimens with balanced safety are a significant advance in vulnerable populations and in resource-limited areas $[15,16]$. Currently, a 3-month once-weekly regimen with rifapentine plus isoniazid (3HP, both with a maximum dose of $900 \mathrm{mg}$ ) is the shortest regimen recommended by the WHO $[5,6]$. In fact, another 3-month regimen with twice-weekly rifapentine plus isoniazid $\left(3 \mathrm{H}_{2} \mathrm{P}_{2}\right.$, both with a maximum dose of $\left.600 \mathrm{mg}\right)$ has been practised in China for years $[17,18]$. However, it had been not been evaluated by randomised controlled trials (RCTs). No study has yet been conducted to evaluate the performance of short-course regimens for LTBI treatment in elderly populations in China or worldwide.

Our aim was to explore optimised LTBI therapy for at-risk subgroups in the elderly under the conditions in China. Our initial design was to evaluate the safety and protective efficacy of two short-course regimens, $3 \mathrm{HP}$ and $2 \mathrm{H}_{2} \mathrm{P}_{2}$ (modified from $3 \mathrm{H}_{2} \mathrm{P}_{2}$; a 2-month twice-weekly regimen of rifapentine plus isoniazid with a maximum dose of $600 \mathrm{mg}$ for each), for selected rural residents aged 50-69 years by means of an RCT.

\section{Methods}

\section{Study regimens}

We conducted an open-label, pragmatic RCT with two intervention groups and one untreated control group. Group A (treated with the $3 \mathrm{HP}$ regimen): 3 months of once-weekly rifapentine (at a dose up to $900 \mathrm{mg}$, with incremental adjustment for subjects weighing $\leqslant 50 \mathrm{~kg}$ ) plus isoniazid (at a dose of 15$25 \mathrm{mg} \cdot \mathrm{kg}^{-1}$ body weight, rounded up to the nearest $50 \mathrm{mg}$, with a maximum dose of $900 \mathrm{mg}$ ). Group B (treated with the $2 \mathrm{H}_{2} \mathrm{P}_{2}$ regimen): 2 months of twice-weekly rifapentine (at a dose of $600 \mathrm{mg}$, with incremental adjustment for subjects weighing $\leqslant 50 \mathrm{~kg}$ ) plus isoniazid (at a dose of $15 \mathrm{mg} \cdot \mathrm{kg}^{-1}$ body weight, rounded up to the nearest $50 \mathrm{mg}$, with a maximum dose of $600 \mathrm{mg}$ ). Group C: untreated controls. Both of the drug regimens were delivered after meals under direct observation. 
Details of the study design are provided in the trial protocol (supplementary material). The trial was registered at the Chinese Clinical Trial Registry with identifier ChiCTR-IOR-15007202.

\section{Ethical review}

The study protocol was approved by the ethics committees of the Institute of Pathogen Biology, Chinese Academy of Medical Sciences, Beijing, China (approval IPB-2015-5). Written informed consent was obtained from all study participants for baseline LTBI testing and other examinations. Considering the limited educational background of the studied rural elderly, we visited eligible participants again for written informed consent before randomisation to guarantee they were clearly aware of their rights and duties in the study. Untreated controls were settled and permitted for the following considerations: 1) this is the first RCT to evaluate the performance of $3 \mathrm{HP}$ in a Chinese population, so no previous data on efficacy and safety was available; and 2) no global or regional guidelines have suggested the elderly as one of the target populations for LTBI management, lacking evidence in benefit-risk analysis for this specific population in worldwide. Proswell Medical Company (Beijing, China) provided independent monitoring during study implementation.

\section{Sample size determination}

The study was designed to assess the protective effectiveness of the studied regimens compared with untreated controls. The protective rate in 2 years was assumed to be $70 \%$ for the two regimens on the basis of an assumed effectiveness of $70 \%$ for isoniazid $[19,20]$. Without treatment, the risk of active TB among this study population in 2 years was estimated to be $2 \%$. Thus, assuming $20 \%$ treatment discontinuation and/or loss to follow-up for the intervention group and $10 \%$ treatment discontinuation and/or loss to follow-up for the control group, we determined that a sample size of 1300 subjects in the intervention group and 1140 subjects in the control group would provide a power of $80 \%$ on the basis of an $\alpha$ level of 0.05 and a two-sided test. We assumed that $20 \%$ of rural residents aged 50-69 years were interferon- $\gamma$ release assay (QuantiFERON-TB Gold In-Tube (QFT); Qiagen, Germantown, MD, USA)-positive, and $90 \%$ of subjects with LTBI would meet the inclusion and exclusion criteria; therefore, a total of 20777 rural residents aged 50-69 years needed testing for LTBI to meet the statistical requirements.

\section{Study population and baseline screening}

The target population was rural residents aged 50-69 years registered in Zhongmu County, an area with a national average epidemic of TB in China. Baseline screening was conducted between July 29, 2015 and December 6, 2015. In short, for each study participant, trained interviewers collected sociodemographic information by use of a standardised questionnaire. Blood samples were collected for QFT testing and other laboratory tests. QFT testing was performed as recommended by the manufacturer using a cut-off value of $\geqslant 0.35 \mathrm{IU} \cdot \mathrm{mL}^{-1}$ for a positive result. Current active or suspected TB was excluded from asymptomatic participants with positive QFT results by digital chest radiography evaluation. Participants with suspected symptoms and/or abnormal chest radiography findings were transferred to the local Center for Diseases Control and Prevention for diagnosis.

All eligible subjects were required to: 1) be 50-69 years old, 2) be local rural residents who would be able to complete the whole study period, 3) have a positive QFT result and 4) voluntarily sign the informed consent form. Exclusion criteria included: 1) confirmed or suspected current active TB; 2) self-reported or registered prior TB; 3) pregnant or lactating females or females preparing for pregnancy; 4) a history of LTBI prophylactic treatment with rifapentine persistently for $>14$ days or with isoniazid intermittently for $>30$ days in the previous 2 years; 5) contraindication for rifapentine or isoniazid; 6) liver dysfunction or impairment (serum alanine aminotransferase (ALT) and/or aspartate aminotransferase (AST) $>3$ times the upper limit of normal (ULN), and/or total bilirubin $>1.5 \times \mathrm{ULN}$, and/or accompanied by liver impairment symptoms and signs); 7) white blood cell count $<2.0 \times 10^{9} \mathrm{~L}^{-1}$; 8) positive for hepatitis B virus (HBV) surface antigen and/or antibody to hepatitis $\mathrm{C}$ virus (HCV) and/or antibody to HIV; 9) renal insufficiency or degeneration; 10) autoimmune diseases or undergoing treatment with immunosuppressive agents; 11) addicted to drinking or actively using street drugs; 12) without capacity to act independently owing to mental disorders or disability; and 13) other conditions inapplicable for participation in this study judged by the investigator, such as failing to cooperate in the test or complete the whole study.

Formal assessment for treatment eligibility included acquiring a second informed consent before the intervention treatment was ended by November 6, 2015.

\section{Randomisation and intervention implementation}

Eligible participants were assigned to study Groups A, B and C according to simple unrestricted randomisation with a sample size ratio of 1:1:0.9 (A:B:C), given a lower loss to follow-up rate in Group C. All participants included in the intervention groups started treatment with the same schedule. 
Information on adverse events and side-effects was self-reported by participants at the time of occurrence or collected by researchers at direct observations. Clinical examinations monitoring side-effects were performed once a month or at a frequency determined by the physicians according to the situation during treatment.

\section{Follow-up examinations and end-points}

Subjects were followed for 24 months after treatment. All participants were evaluated at 3 months after treatment to monitor long-term side-effects. In addition, study follow-up for active case finding occurred quarterly by TB symptom review through door-to-door or telephone surveys and yearly by chest radiography screening.

The primary study end-points were microbiologically confirmed active pulmonary $\mathrm{TB}$ or clinically determined pulmonary TB. Individuals with positive results by sputum smear, culture and/or GeneXpert MTB/RIF assay (Cepheid, Sunnyvale, CA, USA) were confirmed as active TB. Response to empiric TB treatment among participants suspected as having TB but negative to microbiological tests was evaluated for a diagnosis of clinical TB by consensus from a panel of experts consisting of four radiologists, two clinical experts and one laboratory expert. TB diagnosis was made by the expert panel blinded to treatment assignment.

Additional secondary end-points were completion of study therapy, permanent discontinuation of therapy and permanent discontinuation because of an adverse drug reaction, any grade 3 or 4 drug-related toxic effects, or death from any cause. Adverse events and side-effects were graded by investigators using the Common Terminology Criteria for Adverse Events (version 4.0). Two physicians, blinded to treatment allocation, co-determined whether the reported side-effect was study drug related.

Individual treatment termination was determined by the physicians based upon clinical needs. To guarantee the safety of the treated elderly, early termination of the therapy for all participants could be considered but should be approved by the ethics committees. Such early termination was determined by conservative criteria based on existing evidence for the 3HP regimen until October 2015 (supplementary material files S1 and S2): 1) occurrence of high-grade events (grade 3 or 4 ) $>5 \%$ and there is still a trend of growth, 2) occurrence of hepatotoxicity (defined by AST/ALT $>3 \times \mathrm{ULN}$ with symptoms or AST/ALT $>5 \times$ ULN) $>1 \%$ and there is still a trend of growth, and 3) any other unexpected condition that might damage the risk-benefit balance in the study population as evaluated by the researchers and ethics committees.

\section{Statistical analysis}

The frequency of categorical variables in the study participants was compared between groups using the Chi-squared test. The intention-to-treat analysis included all enrolled eligible subjects in each group. The per-protocol population included all eligible enrolled subjects who completed the assigned study regimen (defined as completed $\geqslant 90 \%$ doses of therapy). Incidence rates of active TB were assessed at 24 months after the completion of therapy. To identify potential variables related to the risk of side-effects, all variables showing significant relations in the univariate analysis were entered into the unconditional multiple logistic regression analyses, and the associations were then assessed by means of odds ratios and 95\% confidence intervals. To identify potential variables related to active disease risk, Cox proportional hazards regression models were fitted to estimate hazard ratios. The time duration for each participant involved in the study was calculated based on the quarterly follow-up records. All variables with $\mathrm{p}<0.05$ in univariate models were entered into the multivariate models.

\section{Results}

\section{Study participants}

As shown in figure 1, a total of 20555 rural residents aged 50-69 years were investigated and 19891 of them completed a physical examination. 4116 participants were identified to be QFT-positive, with a prevalence of $20.69 \%$ (4116 out of 19891). During study participant enrolment, 378 QFT-positive individuals were excluded according to the exclusion criteria and 3738 subjects were included for randomisation.

After randomisation, the 3738 eligible participants were classified into three groups: 1284 in Group A (for treatment with the $3 \mathrm{HP}$ regimen), 1299 in Group B (for treatment with the $2 \mathrm{H}_{2} \mathrm{P}_{2}$ regimen) and 1155 in Group C (untreated controls). The clinical and demographic characteristics of the intention-to-treat population are shown in table 1. Of the 3738 included eligible participants, 2054 (54.95\%) were males, $1522(40.72 \%)$ had a current or past history of smoking, 281 (7.52\%) had diabetes based on fasting blood glucose $>7 \mathrm{mmol} \cdot \mathrm{L}^{-1}$ and $64(1.71 \%)$ were identified with pulmonary fibrotic lesions by chest radiography. 
All of the variables were evenly distributed, except for significantly less pulmonary fibrotic lesions in Group B (1.08\%) compared with Group C (2.42\%).

\section{Early termination of treatment}

Both of the regimens were terminated early due to the unexpected high frequency of side-effects (supplementary files S1 and S2). As approved by the ethic committees, the course of regimen A was modified to 8 weeks (between November 7, 2015 and January 2, 2016) and the course of regimen B was modified to 6 weeks (between November 25, 2015 and January 2, 2016). As shown in table 2, the completion rate of modified regimen A was $85.20 \%$ (1094 out of 1284 ), with $40.53 \%$ (77 out of 190) discontinuing treatment due to side-effects. The completion rate of modified regimen B was $78.06 \%$ (1014

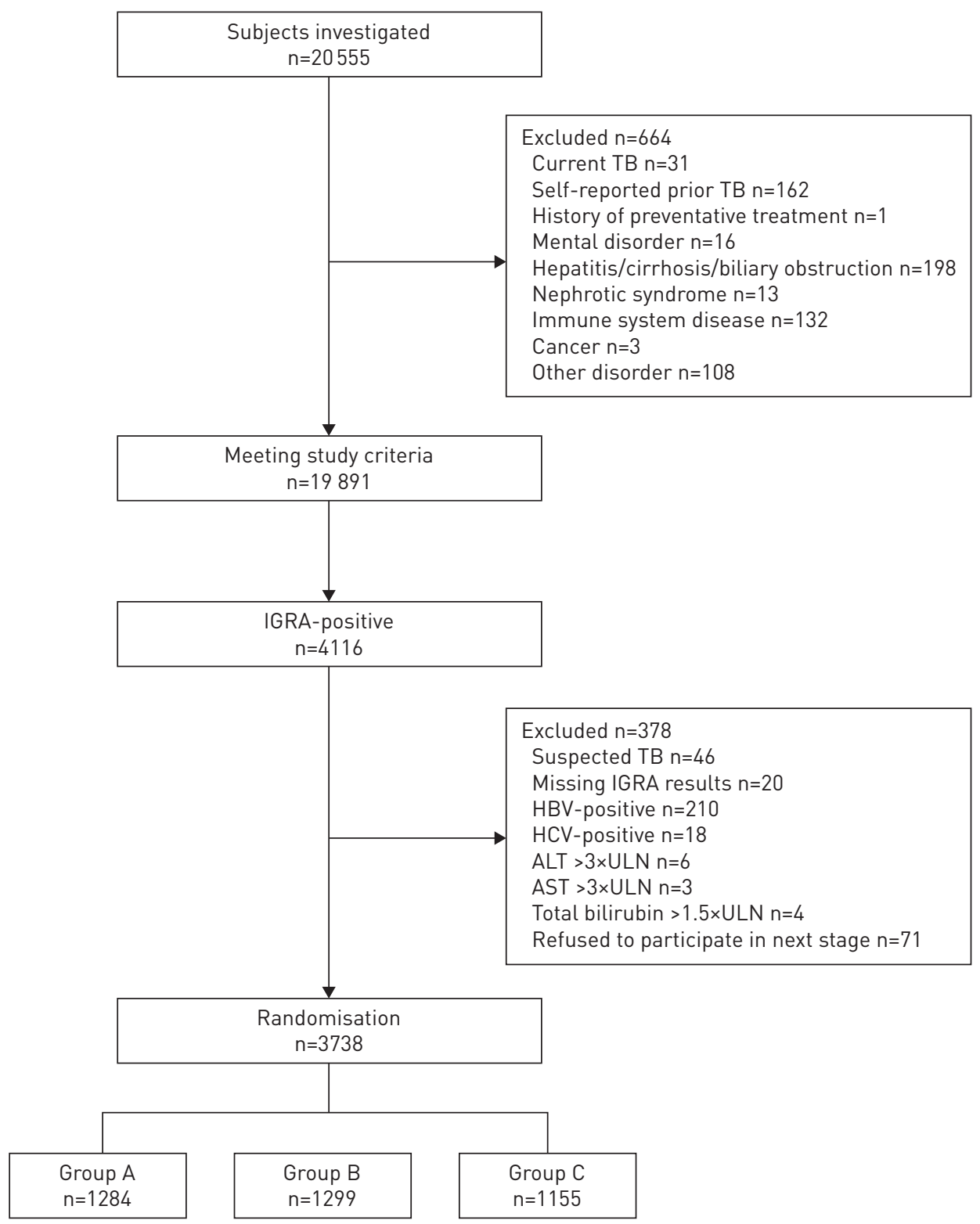

FIGURE 1 Flowchart of study participant enrolment and randomisation. TB: tuberculosis; IGRA: interferon- $\gamma$ release assay; HBV: hepatitis B virus; HCV: hepatitis C virus; ALT: alanine aminotransferase; ULN: upper limit of normal; AST: aspartate aminotransferase. A total of 20555 rural residents aged 50-69 years were investigated and 19891 of them completed a physical examination. 4116 participants were identified to be IGRA (QuantiFERON-TB Gold In-Tube)-positive; 378 of them were excluded according to exclusion criteria and 3738 were included for randomisation. Finally, participants were classified into three groups with a sample size ratio of 1:1:0.9 (A:B:C), given a lower loss to follow-up rate in Group C. 
TABLE 1 Characteristics of the study participants included in the intention-to-treat analysis

\begin{tabular}{|c|c|c|c|c|}
\hline & Group $A^{\#}$ & Group B" & Group $\mathrm{C}^{+}$ & Total \\
\hline Subjects & 1284 & 1299 & 1155 & 3738 \\
\hline Male & 703 (54.75) & 709 (54.58) & $642(55.58)$ & $2054(54.95)$ \\
\hline \multicolumn{5}{|l|}{ Age years } \\
\hline $50-55$ & $329(25.62)$ & $317(24.40)$ & $265(22.94)$ & $911(24.37)$ \\
\hline $56-60$ & $332(25.86)$ & $344(26.48)$ & $295(25.54)$ & 971 (25.98) \\
\hline $61-65$ & 347 (27.02) & $333(25.64)$ & $302(26.15)$ & $982(26.27)$ \\
\hline $66-69$ & $276(21.50)$ & $305(23.48)$ & $293(25.37)$ & 874 (23.38) \\
\hline \multicolumn{5}{|l|}{$\mathrm{BMI} \mathbf{k g} \cdot \mathrm{m}^{-2}$} \\
\hline$<18.5$ & $37(2.88)$ & $30(2.31)$ & $22(1.90)$ & 89 (2.38) \\
\hline $18.5-<24$ & 523 (40.73) & $543(41.80)$ & $472(40.87)$ & $1538(41.14)$ \\
\hline $24-<28$ & $517(40.26)$ & 493 (37.95) & 459 (39.74) & 1469 (39.30) \\
\hline$\geqslant 28$ & $207(16.12)$ & $233(17.94)$ & $202(17.49)$ & $642(17.17)$ \\
\hline Completed primary school & 721 (56.20) & 734 (56.59) & 635 (57.89) & $2090(55.91)$ \\
\hline Married & $1273(99.14)$ & 1289 (99.23) & $1146(99.22)$ & $3708(99.20)$ \\
\hline Household per capita income >RMB6000 & $678(52.80)$ & $655(50.42)$ & 607 (52.55) & $1940(51.90)$ \\
\hline Ever-smoker & $526(40.97)$ & $534(41.11)$ & $462(40.00)$ & $1522(40.72)$ \\
\hline Current alcohol drinker & 403 (31.39) & $398(30.64)$ & $342(29.61)$ & 1143 (30.58) \\
\hline Pulmonary fibrotic lesions & $22(1.71)$ & $14(1.08)^{\S}$ & $28(2.42)$ & $64(1.71)$ \\
\hline Fasting blood glucose $>7 \mathrm{mmol} \cdot \mathrm{L}^{-1}$ & $92(7.17)$ & $87(6.70)$ & $102(8.83)$ & $281(7.52)$ \\
\hline History of silicosis & $31(2.41)$ & $28(2.16)$ & $24(2.08)$ & $83(2.22)$ \\
\hline
\end{tabular}

Data are presented as $\mathrm{n}$ or $\mathrm{n}(\%)$. BMI: body mass index. ${ }^{\#}$ : intervention Group A completed 8 weeks of once-weekly rifapentine plus isoniazid (both with a maximum dose of $900 \mathrm{mg}$ ); ${ }^{\text {१: }}$ intervention Group B completed 6 weeks of twice-weekly rifapentine plus isoniazid (both with a maximum dose of $600 \mathrm{mg}$ ); ${ }^{+}$: blank controls untreated; ${ }^{\S}$ : p-value for Chi-squared test $<0.05$ between Group B and Group C.

out of 1299), with $28.77 \%$ (82 out of 285) discontinuing treatment due to side-effects. More detailed information on therapy completion is given in supplementary material file S3.

\section{Protective effect of the modified regimens}

In total, 30 incident cases of active pulmonary $\mathrm{TB}$ were diagnosed during the follow-up (supplementary material file S4). Supplementary material file S5 shows the Kaplan-Meier curve of time to active TB by study arm. Two cases were confirmed microbiologically, while 28 cases were diagnosed clinically based on their responses to anti-TB treatment. In the intention-to-treat analysis (table 3), the cumulative incidence

\section{TABLE 2 Completion of the modified regimens and attribution for treatment discontinuation}

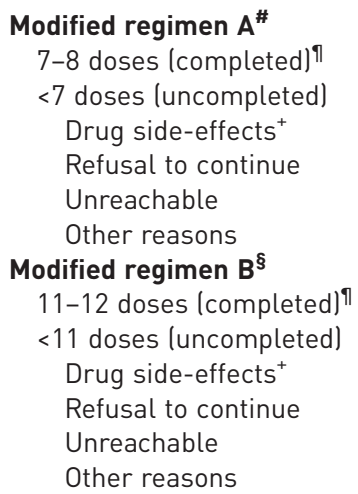

Data are presented as $\mathrm{n}$ or $\mathrm{n}(\%) .{ }^{\#}$ : intervention Group A completed 8 weeks of once-weekly rifapentine plus isoniazid (both with a maximum dose of $900 \mathrm{mg}$ ); ๆ: defined as completed $\geqslant 90 \%$ doses of therapy, i.e. completed $\geqslant 7$ doses for modified regimen $A$ and $\geqslant 11$ doses for modified regimen $B ;{ }^{+}$: side-effects were identified and graded by physicians blinded to treatment allocation according to the Common Terminology Criteria for Adverse Events (version 4.0); ${ }^{\S}$ : intervention Group B completed 6 weeks of twice-weekly rifapentine plus isoniazid (both with a maximum dose of $600 \mathrm{mg}$ ). 
TABLE 3 Incidence of active tuberculosis in the study groups

Group A treated with modified regimen $A^{\#}$
Group B treated with modified regimen $B^{\text {T }}$
Group $C$ untreated

Intention-to-treat analysis
Subjects n
Person-years n
Incident cases n
Cumulative incidence $(95 \% \mathrm{CI}) \%$
Protective rate $\%$
Incidence rate per 100 person-years $(95 \% \mathrm{CI})$
Protective rate $\$$
Adjusted hazard ratio $(95 \% \mathrm{CI})^{f}$
Per-protocol analysis
Subjects n
Person-years n
Incident cases $\mathrm{n}$
Cumulative incidence $(95 \% \mathrm{CI}) \%$
Protective rate $\%$
Incidence rate per 100 person-years $(95 \% \mathrm{CI})$
Protective rate $\$$
Adjusted hazard ratio $(95 \% \mathrm{Cl})^{f}$

1284
2419
10
$0.78(0.30-1.26)$
35.54
$0.41(0.21-0.74)$
39.71
$0.63(0.27-1.44)$

1093
2087
9
$0.82(0.29-1.36)$
32.23
$0.43(0.21-0.79)$
36.76
$0.64(0.27-1.50)$

1299
2471
6
$0.46(0.17-1.00)$
61.98
$0.24(0.14-0.51)$
64.71
$0.41(0.15-1.08)$
1015
1951
4
$0.39(0.10-0.78)$
67.77
$0.21(0.07-0.49)$
69.12
$0.34(0.11-1.06)$

1155

2057

14

$1.21(0.58-1.84)$

$0.68(0.38-1.12)$

Reference

1155

2057

14

$1.21(0.58-1.84)$

$0.68(0.38-1.12)$

Reference

\#: 8 weeks of once-weekly rifapentine lat a maximum dose of $900 \mathrm{mg}$ ) plus isoniazid (at a maximum dose of $900 \mathrm{mg}$ ): ๆ: 6 weeks of twice-weekly rifapentine (at a maximum dose of $600 \mathrm{mg}$ ) plus isoniazid lat a maximum dose of $600 \mathrm{mg}$ ); ${ }^{+}$: calculating protective rate by using cumulative incidence; ${ }^{\S}$ : calculating protective rate by using incidence rate per 100 person-years; ${ }^{f}$ : adjusted for age, sex, body mass index, current alcohol drinking and presence of pulmonary fibrotic lesions.

of active TB was $1.21 \%$ (95\% CI $0.58-1.84 \%$ ) in the untreated control Group C, $0.78 \%$ (95\% CI 0.30 $1.26 \%$ ) in the modified Group A and $0.46 \%$ (95\% CI $0.17-1.00 \%)$ in the modified Group B. Compared with the untreated controls, the risk of active disease was decreased among individuals treated with modified regimen A (adjusted HR 0.63, 95\% CI 0.27-1.43) and modified regimen B (adjusted HR 0.41, 95\% CI $0.15-1.09$ ). In the per-protocol analysis, the protection rates were $36.76 \%$ for modified regimen $\mathrm{A}$ and $69.12 \%$ for modified regimen $\mathrm{B}$.

Among a total of 475 individuals (190 in Group A and 285 in Group B) who did not complete the modified regimens, three of them (one in Group A and two in Group B) developed active TB during the 2 years of follow-up, with a cumulative incidence of $0.63 \%$. Among 320 individuals with LTBI but excluded before randomisation, five of them developed active TB during the 2 years of follow-up, with a cumulative incidence of $1.56 \%$.

\section{Adverse events}

A total of 29 deaths occurred during the study period in the three groups. No significant difference in mortality was observed between the groups $(\mathrm{p}=0.245)$ and none of the deaths were attributed to LTBI treatment (supplementary material file S6). As shown in table 4, there was no significant difference between the intervention groups in the occurrence of any adverse or serious adverse events. For both groups, the occurrence of symptomatic liver injury and abnormal liver function (ALT/AST) was remarkably increased after 4 weeks of treatment (supplementary material file S7). The proportion of subjects with hepatotoxicity was similar between the two treatment groups $(1.02 \%$ in Group A versus $1.17 \%$ in Group B; $\mathrm{p}=0.704)$. Occurrences of other study drug-related side-effects are given in detail in supplementary material files S8 and S9. In short, modified regimen A showed significantly higher proportions of gastrointestinal reactions $(8.60 \% ; \mathrm{p}=0.006)$ and influenza-like symptoms $(3.60 \% ; \mathrm{p}=0.046)$ compared with modified regimen $\mathrm{B}(5.16 \%$ and $2.27 \%$, respectively). A much higher frequency of hypersensitivity or allergy was observed for modified regimen B $(5.08 \%)$ than modified regimen A $(3.36 \%$; $\mathrm{p}=0.031$ ). No significant difference in hospitalisation due to side-effects was found between the two regimens (supplementary material file S10).

All side-effects recovered after terminating treatment or giving clinical management. Monitoring the long-term effects of preventative therapies, a total of seven subjects were identified with elevated ALT/AST $>3 \times \mathrm{ULN}$, but none of them had $>5 \times \mathrm{ULN}$ and no renal dysfunction was identified. 
TABLE 4 Characteristics of the participants with adverse events and study drug side-effects

\begin{tabular}{|c|c|c|c|}
\hline & Group $A^{\#}$ & Group B & p-value for Chi-squared test \\
\hline Subjects & 1279 & 1279 & \\
\hline \multicolumn{4}{|l|}{ Adverse events ${ }^{+}$} \\
\hline Subjects with any adverse event & $295(23.06)$ & $277(21.66)$ & 0.393 \\
\hline Death & $1(0.08)$ & $0(0)$ & 0.999 \\
\hline \multicolumn{4}{|l|}{ Occurrence of serious adverse events } \\
\hline Yes & $18(1.41)$ & $17(1.33)$ & 0.865 \\
\hline No & $277(21.66)$ & $260(20.33)$ & 0.409 \\
\hline \multicolumn{4}{|l|}{ Frequency per person } \\
\hline 1 event & 265 (20.72) & 248 (19.39) & 0.401 \\
\hline$>1$ event & $30(2.35)$ & 29 (2.27) & 0.895 \\
\hline \multicolumn{4}{|l|}{ Attributed to drug } \\
\hline Yes & $244(19.08)$ & 219 (17.12) & 0.199 \\
\hline No & $51(3.99)$ & $58(4.53)$ & 0.493 \\
\hline \multicolumn{4}{|l|}{ Classified by event severity grade } \\
\hline Grade 1 & $212(16.58)$ & 182 (14.23) & 0.100 \\
\hline Grade 2 & $51(3.99)$ & $63(4.93)$ & 0.250 \\
\hline Grade 3 & $30(2.35)$ & $32(2.50)$ & 0.797 \\
\hline Grade $4 / 5$ & $3(0.23)$ & $1(0.08)$ & 0.625 \\
\hline \multicolumn{4}{|l|}{ Side-effects ${ }^{+}$} \\
\hline Subjects with any side-effect & $244(19.08)$ & $219(17.12)$ & 0.199 \\
\hline \multicolumn{4}{|l|}{ Specific side-effect } \\
\hline Gastrointestinal reaction & $110(8.60)$ & $66(5.16)$ & 0.006 \\
\hline Hypersensitivity or allergy & $43(3.36)$ & $65(5.08)$ & 0.031 \\
\hline Influenza-like symptoms $\S$ & $46(3.60)$ & $29(2.27)$ & 0.046 \\
\hline Hepatotoxicity ${ }^{f}$ & $13(1.02)$ & $15(1.17)$ & 0.704 \\
\hline Other drug reactions & $116(9.07)$ & $111(8.68)$ & 0.728 \\
\hline \multicolumn{4}{|l|}{ Categories occurring per person } \\
\hline 1 & $177(13.84)$ & $163(12.74)$ & 0.415 \\
\hline 2 & $53(4.14)$ & 45 (3.52) & 0.410 \\
\hline$\geqslant 3$ & $14(1.09)$ & $11(0.86)$ & 0.547 \\
\hline Hospitalisation due to side-effects & $4(0.31)$ & $6(0.47)$ & 0.753 \\
\hline
\end{tabular}

Data are presented as $\mathrm{n}$ or $\mathrm{n}(\%)$, unless otherwise stated. ULN: upper limit of normal. " : completed 8 weeks of once-weekly rifapentine plus isoniazid (both with a maximum dose of $900 \mathrm{mg}$ ); ": completed 6 weeks of twice-weekly rifapentine plus isoniazid (both with a maximum dose of $600 \mathrm{mg}$ ); ${ }^{+}$: adverse events and side-effects were identified and graded by physicians using the Common Terminology Criteria for Adverse Events (version 4.0); §: influenza-like symptoms include chills, hyperpyrexia, headache, asthma, dyspnoea, generalised soreness, arthralgia, etc.; ${ }^{f}$ : elevations of serum alanine aminotransferase and/or aspartate aminotransferase $>3 \times$ ULN with accompanying symptoms or $>5 \times$ ULN without symptoms.

\section{Risk factors}

The multiple logistic regression analysis (table 5) indicated that male sex was associated with a lower risk of side-effects than female sex (adjusted OR 0.73, 95\% CI 0.53-0.99). The risk of side-effects decreased with an increase of body mass index (BMI) (adjusted OR 0.94, 95\% CI 0.91-0.97), with the highest proportion of side-effects (23.88\%) observed among those with BMI $<18.5 \mathrm{~kg} \cdot \mathrm{m}^{-2}$ and the lowest $(15.27 \%)$ among those with BMI $\geqslant 28 \mathrm{~kg} \cdot \mathrm{m}^{-2}$. The risk of side-effects increased among the participants taking concomitant drugs (adjusted OR 2.12, 95\% CI 1.65-2.71).

As shown in supplementary material file S11, the incidence of active TB increased along with age in a dose-response relationship. Participants with baseline BMI $<18.5 \mathrm{~kg} \cdot \mathrm{m}^{-2}$ had a much higher hazard of developing active disease (adjusted HR 3.15, 95\% CI 1.01-9.77). Current alcohol drinking was found to be related to declining risk of disease. After excluding self-reported or registered TB patients, individuals with pulmonary fibrotic lesions identified by chest radiography were at a much higher risk of developing active TB compared with those with normal chest radiography (adjusted HR 6.77, 95\% CI 2.29-19.97).

\section{Discussion}

This is the first pragmatic RCT from China addressing LTBI treatment among individuals aged $>50$ years. Neither 3HP (3 months of once-weekly rifapentine plus isoniazid, both with a maximum dose of $900 \mathrm{mg}$ ) nor $2 \mathrm{H}_{2} \mathrm{P}_{2}$ (2 months of twice-weekly rifapentine plus isoniazid, both with a maximum dose of $600 \mathrm{mg}$ ) 


\begin{tabular}{|c|c|c|c|}
\hline & $\begin{array}{c}\text { Proportion with } \\
\text { side-effects }^{\text {I }} n / N(\%)\end{array}$ & $\begin{array}{c}\text { p-value for } \\
\text { Chi-squared test }\end{array}$ & $\begin{array}{l}\text { Adjusted OR } \\
\text { (95\% CI) }\end{array}$ \\
\hline \multicolumn{4}{|l|}{ Age years } \\
\hline$\leqslant 60$ & 219/1273 (17.20) & 0.039 & Reference \\
\hline$>60$ & 244/1193 (20.45) & & $1.20(0.97-1.47)$ \\
\hline \multicolumn{4}{|l|}{ Sex } \\
\hline Female & $238 / 1121(21.23)$ & 0.004 & Reference \\
\hline Male & $225 / 1345$ (16.73) & & $0.73(0.53-0.99)$ \\
\hline \multicolumn{4}{|l|}{ BMI $\mathrm{kg} \cdot \mathrm{m}^{-2}$} \\
\hline$<18.5$ & 16/67 (23.88) & 0.059 & \\
\hline $18.5-<24$ & $211 / 1019$ (20.72) & & \\
\hline $24-<28$ & $172 / 961(17.90)$ & & \\
\hline$\geqslant 28$ & $64 / 419(15.27)$ & & \\
\hline ptrend & & 0.014 & $0.94(0.91-0.97)$ \\
\hline \multicolumn{4}{|c|}{ Ever-smoker } \\
\hline No & 293/1457 (20.11) & 0.041 & Reference \\
\hline Yes & $170 / 1007$ (16.85) & & $0.99(0.71-1.38)$ \\
\hline \multicolumn{4}{|c|}{ Current alcohol drinking } \\
\hline No & $327 / 1701$ (19.22) & 0.395 & \\
\hline Yes & $136 / 765(17.78)$ & & \\
\hline \multicolumn{4}{|c|}{ Pulmonary fibrotic lesions } \\
\hline No & $451 / 2416(18.67)$ & 0.068 & \\
\hline Yes & $11 / 36(30.56)$ & & \\
\hline \multicolumn{4}{|c|}{ Fasting blood glucose $\mathrm{mmol} \cdot \mathrm{L}^{-1}$} \\
\hline$\leqslant 7$ & 434/2295 (18.91) & 0.528 & \\
\hline$>7$ & $29 / 171(16.96)$ & & \\
\hline \multicolumn{4}{|c|}{ History of silicosis } \\
\hline No & 449/2409 (18.64) & 0.257 & \\
\hline Yes & $14 / 57(24.56)$ & & \\
\hline \multicolumn{4}{|c|}{ Concomitant with other drugs } \\
\hline No & $346 / 2066$ (16.75) & $<0.001$ & Reference \\
\hline Yes & $117 / 400(29.25)$ & & $2.12(1.65-2.71)$ \\
\hline
\end{tabular}

BMI: body mass index. " : side-effects were identified and graded by physicians using the Common Terminology Criteria for Adverse Events (version 4.0); ": the events in the two intervention groups were pooled for this analysis.

was completed as scheduled due to the increasingly rapid growth and unexpected high frequency of drug-related side-effects. Nevertheless, in the 2 years of follow-up for the modified regimens, the 6-week twice-weekly regimen still showed a protective efficacy $>60 \%$.

Treatment of LTBI can effectively reduce the risk of active TB development with an efficacy ranging from $60 \%$ to $90 \%$ and has long been considered an essential component of TB control in low-incidence countries [21]. Since 2015, the WHO has called for the global goal to end the TB epidemic [4] by strengthening and expanding preventative treatment to high-risk groups as a means to accelerate the decline in the incidence of TB $[5,6]$. Preventative therapy in the elderly with LTBI was suggested to be one effective tool to accelerate the decline in the incidence of TB in China $[12,13]$. However, data on safety and efficacy of LTBI therapies using domestic drugs in the elderly Chinese are lacking. Our results provide direct evidence that the risks of $3 \mathrm{HP}$ are considerable among the elderly. The hepatotoxicity rate of $1 \%$ is 2.5 times the rate observed in the landmark trial on 3HP [19] and the rate of hospitalisation was $0.3 \%, 3$ times higher than reported [22]. It might be attributed to a lower tolerance to anti-TB drugs in the elderly $[15,16]$. Recently, more results of the application of $3 \mathrm{HP}$ in various populations have been reported [23-25]. Rates of hepatotoxicity among patients taking 3HP were lower than comparators in all RCTs. However, it is still difficult to extract safety data for the elderly from these heterogeneous studies [26]. In a small RCT from Taiwan, 1.5\% (two out of 132) of participants undergoing 3HP treatment reported clinically relevant hepatotoxicity [27]. This was the first safety data for 3HP from a Chinese population, but it was achieved from a young population with a mean age of 32 years. In addition, compared with foreign drugs, the varied performance of domestic drugs might be another possible explanation. Unfortunately, no foreign rifapentine and isoniazid are currently approved in China. The fast-growing occurrence of self-reported symptoms and/or laboratory-confirmed hepatotoxicity 
(supplementary material file S7) raises concerns for even higher rates if therapy had been allowed to continue $[28,29]$. Our initially designed $2 \mathrm{H}_{2} \mathrm{P}_{2}$ regimen was adapted from the 3-month twice-weekly rifapentine plus isoniazid regimen $\left(3 \mathrm{H}_{2} \mathrm{P}_{2}\right.$, both with a maximum dose of $\left.600 \mathrm{mg}\right)$ [17, 18]. A non-RCT has been conducted for tuberculin skin test-positive college students using $3 \mathrm{H}_{2} \mathrm{P}_{2}$ (1948 treated and 1765 untreated); the occurrence of liver dysfunction (ALT/AST higher than normal level) was reported to be $2 \%$ and the protective rate was observed to be $75 \%$ during 4 years of follow-up [17]. Compared with $3 \mathrm{HP}$, $3 \mathrm{H}_{2} \mathrm{P}_{2}$ was determined mainly based on the following two points. 1) It was reported that the genetic background, such as drug-metabolising enzyme gene polymorphisms, may contribute to various risks of anti-TB drug-induced liver injury (e.g. the cytochrome P450 $2 \mathrm{E} 1$ genotype $C Y P 2 E 1{ }^{*} 1 \mathrm{~A} /{ }^{\star} 1 \mathrm{~A}$ has been found to be associated with lower tolerance to anti-TB drugs in East Asians [30]). 2) Asians have been reported to carry a higher frequency of rapid acetylator genotypes of $N$-acetyltransferase $2(\sim 50 \%)$ than Caucasians $(\sim 5 \%)$. $N$-acetyltransferase 2 is the major drug-metabolising enzyme of isoniazid and people are identified as rapid or slow acetylators according to its activity. Rapid acetylators are prone to treatment failure, probably due to insufficient exposure to isoniazid [31]. Thus, reduced single dosage and increased frequency were adopted in $3 \mathrm{H}_{2} \mathrm{P}_{2}$. In our trial, $3 \mathrm{H}_{2} \mathrm{P}_{2}$ was shortened to $2 \mathrm{H}_{2} \mathrm{P}_{2}$ at initial study design to improve the tolerance of the elderly.

Efficacy evaluations were implemented to the early terminated regimens, which were modified to a 8-week once-weekly regimen and a 6-week twice-weekly regimen, respectively. This is the first trial to provide safety and efficacy data for such short-course regimens based on rifapentine and isoniazid in the elderly from the general population. It is noteworthy that the 6-week regimen showed a protective efficacy $>60 \%$, which was twice the efficacy observed for the 8-week once-weekly regimen. This provides a positive outlook for the development of a shorter treatment regimen than is currently recommended for the elderly with LTBI. Although the pooled dosages of the two regimens were equal $(7200 \mathrm{mg})$ and the risks of side-effects were also similar, the twice-weekly regimen showed better efficacy and proved to be more suitable for the elderly Chinese population. Research on the pharmacokinetics of the drugs in special populations is warranted to better define an innovative treatment regimen with optimal doses and dosing schedules.

Although the 6-week twice-weekly regimen showed better protection, the completion rate was lower than for the 8 -week regimen ( 8 doses) $(78.06 \%$ versus $85.20 \%$; $\mathrm{p}<0.001)$. The most frequent reason for early termination was refusal to continue $(34.39 \%)$ rather than side-effects $(28.77 \%)$ in the 6 -week regimen. The total number of doses and the frequency of drug delivery seemed to significantly influence LTBI therapy adherence. In March 2018, Richard Chaisson (Johns Hopkins University, Baltimore, MD, USA) shared results of the ACTG 5279 phase 3 trial (ClinicalTrials.gov identifier NCT01404312) at the 2018 Conference on Retroviruses and Opportunistic Infections (Boston, MA, USA). This trial found that the 1-month rifapentine plus isoniazid regimen was noninferior to 9-month isoniazid in HIV-infected adults and adolescents. More recently, an open-label trial conducted in nine countries reported the 4-month regimen of rifampin was noninferior to 9-month isoniazid for the prevention of active TB [32]. Such short-course or ultrashort-course therapies showed fewer adverse events and higher treatment completion, and were suggested to be important tools to control the TB epidemic.

We did not find a relationship between the baseline elevations of AST/ALT and the occurrence of hepatotoxicity during the treatments. Nearly all of the participants (47 out of 48) had asymptomatic marked elevations in liver function tests (AST/AST $>3 \times \mathrm{ULN}$ ). None of the patients with hepatotoxicity were identified in the first month of treatment and severe liver injuries occurred quickly later in the course of therapy (supplementary material file S4). Therefore, baseline and liver function monitoring is needed throughout LTBI treatment among the elderly, especially those with drinking habits.

Individuals with underlying liver disease from $\mathrm{HBV} / \mathrm{HCV}$ infection and viral hepatitis were excluded from our study and cannot explain the high rate of drug-induced hepatotoxicity [19, 33]. However, HBV infection is still common in China, especially in the elderly [34], and may increase the rates of hepatotoxicity. Therefore, future studies are needed to address this viral comorbidity in high-burden regions.

Our study has several other limitations. First, although different from the Peto-Haybittle rule for stopping trials early for lack of evidence of benefit $[35,36]$, our study terminated the designed treatment early due to side-effects and the precision of the outcome estimates for the modified regimen based on the original study settings might also be biased $[37,38]$. For example, for the 6-week twice-weekly regimen, our study only has a power of $54 \%$ to detect a protective rate of $62 \%$ at the significance level of 0.05 (95\% confidence level). Therefore, further RCTs are warranted to systematically evaluate the efficacy of this ultrashort-course regimen and our study provided essential data for the study design of such trials. Second, the 2-year follow-up limits the assessment of long-term protection of the study regimens. Our 
previous study found the elderly in rural China had a higher risk of TB infection acquisition [10], which might shorten the protection period of the treatment compared with settings with a lower risk of TB transmission. This information would be very important for strategic policy development and, hence, we will maintain the study cohorts for longer-term evaluation. Third, study participants with pulmonary fibrotic lesions were not evenly distributed between the groups, which can influence the estimation of protection because prior TB is a risk factor for disease development among individuals with LTBI [7]. However, after excluding participants with pulmonary fibrotic lesions, the protective efficacy of the 6-week regimen was still $47.19 \%$ (supplementary material file S12). Fourth, we observed fewer active TB cases than expected. This might be partly explained by the strict exclusion criteria by which some individuals with risk factors for disease were excluded, thereby potentially creating a bias toward higher protection rates found in the study. Additionally, strict exclusion criteria limited the applicability of our findings in the excluded individuals. Fifth, most of the incident cases (28 out of 30 ) were clinically diagnosed by active case finding in this study. However, we used an expert team to determine clinical diagnosis, blinded to group assignment, based on responses to diagnostic anti-TB treatment. This guaranteed the objectivity and traceability of diagnosis, and minimised the bias caused by misclassification. Nevertheless, there is a need to increase the positive detection rate of microbiological tests, for one, by improving sputum quality.

In conclusion, while the short LTBI regimens tested had fairly high efficacy and look promising, high rates of adverse effects led to early termination of both arms. Thus, these regimens must be used with caution among the elderly. The 6-week twice-weekly rifapentine plus isoniazid regimen showed a protective efficacy $>60 \%$ in our study population. Such innovative regimens with ultrashort courses and optimised use of drugs are urgently needed, especially in high-burden countries, for the global plan of the End TB Strategy. Our findings indicate ultrashort LTBI treatment for at-risk subgroups might be feasible and practicable, but needs further tests in varied populations.

Acknowledgements: We appreciate the assistance of Lixia Wang and Shuigao Jin (Chinese Center for Disease Prevention and Control, Beijing, China), and Yude Chen (Peking University, Beijing, China) for their help with study implementation and data analysis. We thank Shiming Cheng and Liya Wan (Chinese Anti-tuberculosis Association, Beijing, China) for their support in study design. We thank all the investigators in the study team for their contributions (a full list of LATENTTB-NSTM study team members is given in supplementary material file S13).

Author contributions: Q. Jin and L. Gao designed the study. L. Gao, J. Liu, S. Pan, H. Xin, H. Zhang, L. Guan and Z. Liu organised the implementation of the study. H. Xin, H. Zhang, H. Li, X. Li, B. Feng, X. Chen, F. Shen and D. Wang did epidemiological investigation and intervention management. X. Li, B. Feng, X. Chen, and H. Li did the QFT test. Y. Chen and W. Cui did side-effect management during treatment. Z. Zhang, Y. Ma, X. Chen, X. Zhou and X. Guan did active disease diagnosis. H. Xin and L. Gao did data management and data analysis. L. Gao and Q. Jin wrote the report. All authors contributed to review and revision, and have seen and approved the final version of manuscript.

Conflict of interest: None declared.

Support statement: This study was funded by the National Science and Technology Major Project of China (2014ZX10003001-001), the Program for Changjiang Scholars and Innovative Research Team in University of China (IRT13007), the CAMS Innovation Fund for Medical Sciences (2016-I2M-1-013), Qi Jin team of Sanming Project of Medicine in Shenzhen (SZSM 201412001) and the Sanming Project of Medicine in Shenzhen (GCZX2015043015340574). Funding information for this article has been deposited with the Crossref Funder Registry.

\section{References}

1 World Health Organization. Global Tuberculosis Report 2017. 2017. www.who.int/tb/publications/global_report/ en/ Date last accessed: July 29, 2018.

2 Houben RM, Dodd PJ. The global burden of latent tuberculosis infection: a re-estimation using mathematical modelling. PLoS Med 2016; 13: e1002152.

3 Comstock GW, Livesay VT, Woolpert SF. The prognosis of a positive tuberculin reaction in childhood and adolescence. Am J Epidemiol 1974; 99: 131-138.

4 Uplekar M, Weil D, Lonnroth K, et al. WHO's new end TB strategy. Lancet 2015; 385: 1799-1801.

5 World Health Organization. Guidelines on the management of latent tuberculosis infection. 2014. www.who.int/ tb/publications/ltbi_document_page/en/ Date last accessed: July 29, 2018.

6 World Health Organization. Latent tuberculosis infection: updated and consolidated guidelines for programmatic management. 2018. www.who.int/tb/publications/2018/latent-tuberculosis-infection/en/ Date last accessed: July 29, 2018.

7 Gao L, Li XW, Liu JM, et al. Incidence of active tuberculosis among individuals with latent tuberculosis infection in rural China: follow-up results of a population-based multicenter prospective cohort study. Lancet Infect Dis 2017; 17: 1053-1061.

8 Jiang Q, Lu L, Wu J, et al. Assessment of tuberculosis contact investigation in Shanghai, China: an 8-year cohort study. Tuberculosis 2018; 108: 10-15.

9 Gao L, Lu W, Bai LQ, et al. Latent tuberculosis infection in rural China: baseline results of a population-based, multicentre, prospective cohort study. Lancet Infect Dis 2015; 15: 310-319.

10 Gao L, Bai LQ, Liu JM, et al. Annual risk of tuberculosis infection in rural China: a population-based prospective study. Eur Respir J 2016; 48: 168-178. 
11 Wang L, Zhang H, Ruan Y, et al. Tuberculosis prevalence in China, 1990-2010; a longitudinal analysis of national survey data. Lancet 2014; 383: 2057-2064.

12 Lin $\mathrm{HH}$, Wang L, Zhang $\mathrm{H}$, et al. Tuberculosis control in China: use of modelling to develop targets and policies. Bull World Health Organ 2015; 93: 790-798.

13 Ministry of Health of the People's Republic of China. [Administrative measures of the prevention and treatment for tuberculosis]. 2013. www.moh.gov.cn/mohzcfgs/s3576/201303/727b973d5ee4403ead07ela2a22928f6.shtml Date last accessed: July 29, 2018.

14 Sorresso DJ, Mehta JB, Harvill LM, et al. Underutilization of isoniazid chemoprophylaxis in tuberculosis contacts 50 years of age and older. A prospective analysis. Chest 1995; 108: 706-711.

15 Smith BM, Schwartzman K, Bartlett G, et al. Adverse events associated with treatment of latent tuberculosis in the general population. CMAJ 2011; 183: E173-E179.

16 Kunst H, Khan KS. Age-related risk of hepatotoxicity in the treatment of latent tuberculosis infection: a systematic review. Int J Tuberc Lung Dis 2010; 14: 1374-1381.

17 Liu YQ, Tu DH, An YS, et al. Tuberculosis control in college students in Beijing: preventive therapy for tuberculosis-infected persons. Chin J Antitubercul 2005; 27: 139-142.

18 Wang LX, Cheng SM, Zhou L. [Handbook of Tuberculosis Chemoprevention and Immunization]. Beijing, People's Medical Publishing House, 2012.

19 Sterling TR, Villarino ME, Borisov AS, et al. Three months of rifapentine and isoniazid for latent tuberculosis infection. N Engl J Med 2011; 365: 2155-2166.

20 Schechter M, Zajdenverg R, Falco G, et al. Weekly rifapentine/isoniazid or daily rifampin/pyrazinamide for latent tuberculosis in household contacts. Am J Respir Crit Care Med 2006; 173: 922-926.

21 Getahun H, Matteelli A, Chaisson RE, et al. Latent Mycobacterium tuberculosis infection. N Engl J Med 2015; 372: $2127-2135$

22 Sterling TR, Moro RN, Borisov AS, et al. Flu-like and other systemic drug reactions among persons receiving weekly rifapentine plus isoniazid or daily isoniazid for treatment of latent tuberculosis infection in the PREVENT tuberculosis study. Clin Infect Dis 2015; 61: 527-535.

23 Lobue PA, Mermin JH. Latent tuberculosis infection: the final frontier of tuberculosis elimination in the USA. Lancet Infect Dis 2017; 17: e327-e333.

24 Martinson NA, Barnes GL, Moulton LH, et al. New regimens to prevent tuberculosis in adults with HIV infection. N Engl J Med 2011; 365: 11-20.

25 Villarino ME, Scott NA, Weis SE, et al. Treatment for preventing tuberculosis in children and adolescents. JAMA Pediatr 2015; 169: 247-255.

26 Pease C, Hutton B, Yazdi F, et al. A systematic review of adverse events of rifapentine and isoniazid compared to other treatments for latent tuberculosis infection. Pharmacoepidemiol Drug Saf 2018; 27: 557-566.

27 Sun HY, Huang YW, Huang WC, et al. Twelve-dose weekly rifapentine plus isoniazid for latent tuberculosis infection: a multicentre randomised controlled trial in Taiwan. Tuberculosis 2018; 111: 121-126.

28 Saukkonen JJ, Cohn DL, Jasmer RM, et al. An official ATS statement: hepatotoxicity of antituberculosis therapy. Am J Respir Crit Care Med 2006; 174: 935-952.

29 Snider DE Jr, Caras GJ. Isoniazid-associated hepatitis deaths: a review of available information. Am Rev Respir Dis 1992; 145: 494-497.

30 Cai Y, Yi J, Zhou C, et al. Pharmacogenetic study of drug-metabolising enzyme polymorphisms on the risk of anti-tuberculosis drug-induced liver injury: a meta-analysis. PLoS One 2012; 7: e47769.

31 Motta I, Calcagno A, Bonora S. Pharmacokinetics and pharmacogenetics of anti-tubercular drugs: a tool for treatment optimization? Expert Opin Drug Metab Toxicol 2018; 14: 59-82.

32 Menzies D, Adjobimey M, Ruslami R, et al. Four months of rifampin or nine months of isoniazid for latent tuberculosis in adults. N Engl J Med 2018; 379: 440-453.

33 Wang NT, Huang YS, Lin MH, et al. Chronic hepatitis B infection and risk of antituberculosis drug-induced liver injury: systematic review and meta-analysis. J Chin Med Assoc 2016; 79: 368-374.

34 Cui Y, Jia J. Update on epidemiology of hepatitis B and C in China. J Gastroenterol Hepatol 2013; 28: Suppl. 1, $7-10$.

35 Haybittle J. Repeated assessment of results in clinical trials of cancer treatment. Br J Radiol 1971; 44: 793-797.

36 Peto R, Pike M, Armitage P. Design and analysis of randomized clinical trials requiring prolonged observation of each patient: introduction. Br J Cancer 1976; 34: 585-612.

37 Wittes J. Stopping a trial early - and then what? Clin Trials 2012; 9: 714-720.

38 Rahman NM, Davies RJ. Relearning an old lesson: stopping trials early. Thorax 2010; 65: 851-853. 